Appendix E: Geochronology data, in Furer, L.C., FehImann, R.H., Taylor, A.M., Self, G.W., and Amoco Oil Co., Data compilation of the 1971 field party, southeast Brooks Range and Fort Yukon, Alaska; Vol 1

Furer, L.C., and Amoco Oil Co.

GMC DATA REPORT 464E

This GMC data report from the Amoco Heritage collection has been made available through funding from the FY2018 USGS National Geological and Geophysical Data Preservation Program, Grant Number G18AP00054. This project report is presented in its original format and has not been reviewed for technical content or for conformity to the editorial standards of DGGS. It should not be used or cited as reviewed data.

2019

State of Alaska

Department of Natural Resources

Division of Geological \& Geophysical Surveys

GEOLOGIC MATERIALS CENTER
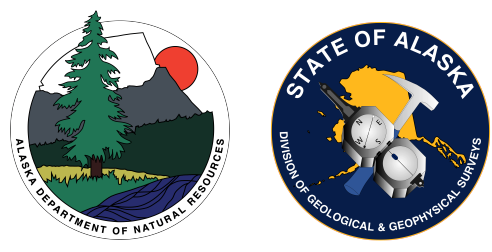



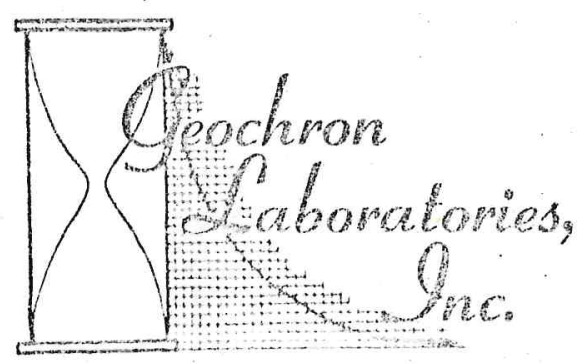

24 Blachstone stree?, Cambidge, Mass, 02139

Telephone Thowbridge 6-3691

4 Jamuary 1972

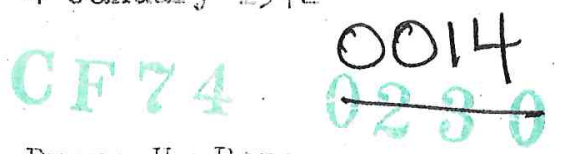

Duane H. Reno

Amoco Production $\mathrm{Co}$

Security Life B]dg.

Denver, Colorado 80202

Dear Mr. Reno:

We have now completed the $K$ ar age detexminations on the seven (7) samples described in your letter of 12 . Hovermer 1971. You will find the complete analytical reports enclosed.

All but one of the samples had to be analyzed as whole rocks; we were able to get a quite good sanidine concentrate from sample HRJm. The samples gave quite a spread of ages, from about 20 million years to 309 million years. The ages of the obviously sheared and altexed rocks should of course be considered to be rainimurn ages of the rocks and probably approach the times of metamorphism and/or alteration. I would expect the ages of HiRI, I and LCF I9 to be close to the true ages of the rocks. Sample 8007 appeared to be a migmatite which contained inclusions of slaty material. We crushed this sample and handpicked severaj. grams of the slate, which was then analyzed as a whole rock. I would expect the age of 8007 to be very close to the age of metamorphism of the original material.

I trust that you will find these reports useful, and if you should have any questions about them please do not hesitate to contact me. In the meantime, I an enclosing our invoice for this work. I hope that we may be able to serve you again in the near future.

Sincerely,

GEOCHRON ILABORATORTMS DIV.
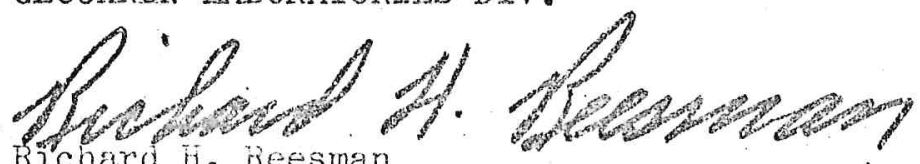

General Vianager

$\mathrm{RHR} / \mathrm{dm}$ 


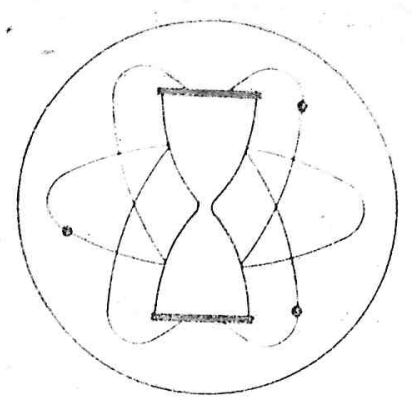

KRUEGER ENTERPRISES, INC.

GEOCHRON LABorATORIES DIVISION

24 BLACKSTONE STREET O CAMBRIDGE, MA. $02139 \cdot(6) 1 \%, 876.369$

Our Sample No. R-2167

Your Reference: 6112

Date Received: 29 November 1.97 .2

Submitred by: Duane H. Reno

Date Reported: 4 January 1972

Amoco Production Co.

Security life Bldg.

Denver, Colorado 80202

Sample Description \& Locality:

Sheared andesite (?)

Northern Alaska

Material Analyzed: Whole rock, crushed to $-40 /+100$ mesh.

$\operatorname{Ar} 40 * / K^{40}=.005837$

$\mathrm{AGE}=97.2 \pm 4.1 \quad$ M.Y.

Argon Analyses:

Ar ${ }^{40 *}$, ppm.

$.0 .112 \%$

.01114

Potassium Analyses:

$\% K$

1.576

1.571

$$
\operatorname{Ar} 40 \% / \operatorname{Total} A r^{40}
$$

.495

.735
Ave. Ar $40 *$, ppm.

.01121

\section{Constants Used:}

$\lambda_{\beta}=4.72 \times 10^{-10} /$ year

$\lambda_{\mathrm{e}}=0.585 \times 10^{-10} /$ year

Ave. \% K

1.573
$\mathrm{K}^{40}, \mathrm{ppm}$

1.919

$K^{40} / K=1.22 \times 10^{-4} \mathrm{~g} . / \mathrm{g}$.

$A G E=\frac{1}{\lambda_{e}+\lambda_{\beta}} \ln \left[\frac{\lambda_{\beta}+\lambda_{e}}{\lambda_{e}} \times \frac{A r^{40 *}}{K^{40}}+1\right]$

Note: $A r^{40 *}$ refers to radiogenic $A r^{40}$.

M.Y. refers to millions of years. 


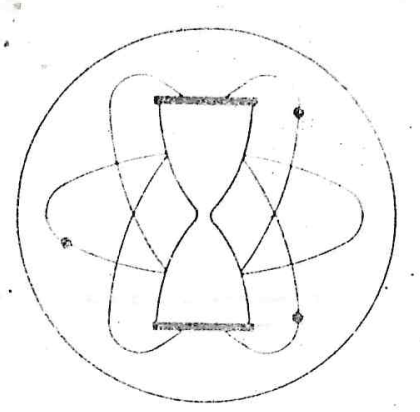

\section{KRUEGER ENTERPRISES, INC. GEOCHRON LABORATORIES DIVISION}

Our Sample No.R-21.64

Date Recoived: 29 Noveraber 1971.

Your Peference: 8007

Date Feported: 4 January 1972

Submited by: Duane H. Reno

Anoco Production Co.

Security Life B1dg.

Denver, Colorado 80202

Sample Description \& Locality:

$$
\begin{aligned}
& \text { Metasediment (?) Metacgl. } \\
& \text { Northexn A.laska }
\end{aligned}
$$

Material Analyzed: S1aty fragments, handpicked from rock, crushed to $-40 /+100$ mesh.
Ar ${ }^{40} * / K^{40}=.007851$
$\mathrm{AGE}=$
$130 \pm 5$
M.Y.

\section{Argon Analyses:}

$\begin{array}{ccc}\text { Ar }{ }^{40 *}, \mathrm{ppm} . & \mathrm{Ar}^{40 *} / \operatorname{Total~Ar}^{40} & \text { Ave. Ar }{ }^{40 *}, \mathrm{ppm} \\ .05185 & .786 & .05123 \\ .05061 & .802 & \end{array}$

Potassium Analyses:

$\% k$

5.361

5.336

Constants Used:

$\lambda_{\beta}=4.72 \times 10^{-10} /$ year

$\lambda_{e}=0.585 \times 10^{-10} /$ year

$K^{40} / K=1.22 \times 10^{-4} \mathrm{~g} . / \mathrm{g}$.

Note: $A r^{40 *}$ refers to radiogenic $\mathrm{Ar}^{40}$.

M.Y. reters to millions of years.
Ave. $\% K$

5.348

$K^{40}$, ppm

6.525 


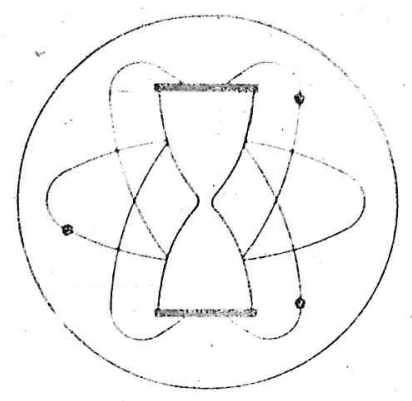

KRUEGER ENTERPRISES, INC.

GEOCHRON LABORATORIES DIVISION

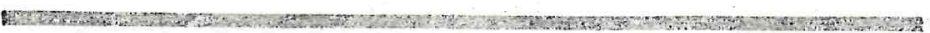

24 BLACKSTONE STREET E CAMBRIDGE, MA. 02139- (617). 876. 3691

Our Sample No. F-2268

Date Received: 29 November 1.971

Your Reference: HRIm].

Date Reported: 4 Januery 1972

Submitred by: Duane Ho Reno

Arroco Production Co.

Security Life Blag.

Denver, Colorado 80202

Sample Description \& Locality:

Altered rhyolite porphyry

Northern Alaska

Material Analyzed: Feldspar concentrate, $-40 /+100$ mesh.

A. ${ }^{40 * / K^{40}}=.003215$

$A G E=54.2 \pm 2.0 \quad$ M.Y.

Argon Analyses:

$\mathrm{Ar}^{40}$, ppm.

.03549

.03592
$\operatorname{Ar}^{40 *} / \operatorname{Total}^{\mathrm{A}} \mathrm{r}^{40}$

.485

.513
Ave. $\mathrm{Ar}^{40 *}, \mathrm{ppm}$

.03571

Potassium Analyses:

$\% k$

9.062

9.144

\section{Constants Usod:}

$\lambda_{\beta}=4.72 \times 10^{-10} /$ year

$\lambda_{\mathrm{e}}=0.585 \times 10^{-10} /$ year

$K^{40} / K=1.22 \times 10^{-4} \mathrm{~g} / \mathrm{g}$.

Note: $\operatorname{Ar}^{40 *}$ refers to radiogenic $A r^{40}$.

M.V. refers to millions of years.
Ave. \%K

9.103

$\mathrm{K}^{40}, \mathrm{ppm}$

11.105
$A G E=\frac{1}{\lambda_{e}+\lambda_{\beta}} \ln \left[\frac{\lambda_{\beta}+\lambda_{e}}{\lambda_{e}} \times \frac{A r^{40 *}}{K^{40}}+1\right]$ 


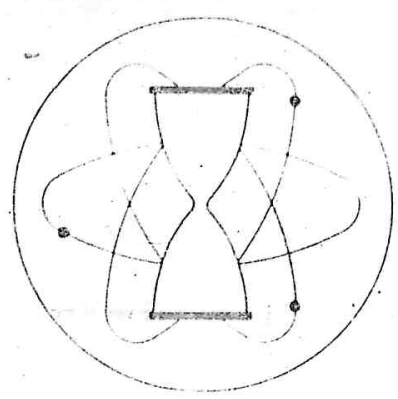

\section{KRUEGER ENTERPRISES, INC. GEOCHRON LABORATORIES DIVISION}

Our Sample No. R-2165

Your Reference: 6229

Submited by: Duane H. Reno Anoco Production Co.

Security Life Bldg.

Denver, Colorado 80202

Sample Description \& Localiny:

Pinylitite.

Northern Alaska

Material Analyzed: Whole rock, crushed to $-40 /+100$ mesh.
$\operatorname{Ar}^{40 * / K^{40}}=.01961$
$A G E=309 \pm 11$
Mi.Y.

Argon Analyses:
$\mathrm{Ar}^{40 *}$,ppm.
$\operatorname{Ar}^{40 *} / \operatorname{Total} \mathrm{Ar}^{40}$
Ave. $A r^{40 *}, p p m$.
.08815
.920
.09161
.925
.08988

Potassium Analyses:

$\% K$

3.702

3.812 .
Ave. \%K

3.757
$K^{40}$, ppm

4.583

Constants Used:

$\lambda_{\beta}=4.72 \times 10^{-10} /$ year

$\lambda_{e}=0.585 \times 10^{-10} /$ year

$$
A G E=\frac{1}{\lambda_{e}+\lambda_{\beta}} \ln \left[\frac{\lambda_{\beta}+\lambda_{e}}{\lambda_{e}} \times \frac{A r^{40 *}}{K^{40}}+1\right]
$$

$K^{40} / K=1.22 \times 10^{-4} \mathrm{~g} . / \mathrm{g}$.

Note: $A r^{40 *}$ refers to radiogenic $A r^{40}$.

M.Y. refers to millions of years. 


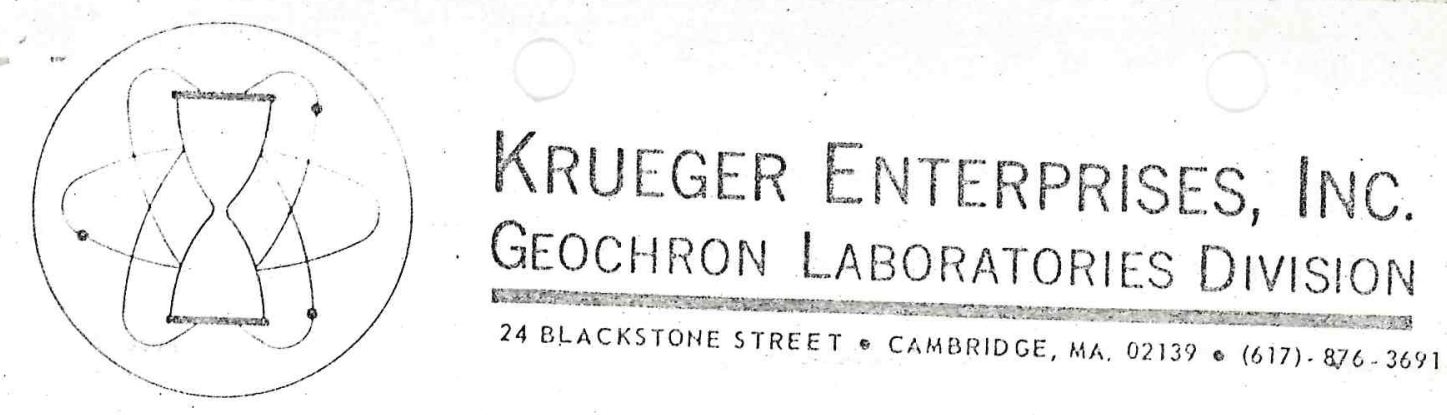

POTASSIUM-ARGON AGE DETERMINATION

REPORT OF ANALYTICAL WORK

Our Sample No. R- 2166

Your Reference: 6053

Date Received: 29 November 1971

Submitied by: Duane H. Reno

Date Reported: 4 January 1972

Amoco Production Co.

Security Ijife Bldg.

Denver, Colorado 80202

Sampla Description \& Locally:

Sheared andesite

Northern Alaska

Material Analyzed: Whole rock, crushed to $-40 /+100$ mesh.
Ar ${ }^{40 *} / K^{40}=.009562$
$A G E=$
$157 \pm 10$
M.Y.

\section{Argon Analyses:}
Ar ${ }^{40 *}$, ppom.
$\operatorname{Ar}^{40 * / T o t a l ~ A r} 40$
Ave. Ar $40 *$, ppm.
.003403
.003538
.342
.375
.003471

Potassium Analyses:

$\% K$

.299

.296
Ave. \%K
$K^{40}, \mathrm{ppm}$

.362

\section{Constants Used:}

$\lambda_{\beta}=4.72 \times 10^{-10} /$ year

$\lambda_{\mathrm{e}}=0.585 \times 10^{-10} /$ year

$A G E=\frac{1}{\lambda_{e}+\lambda_{\beta}} \ln \left[\frac{\lambda_{\beta}+\lambda_{e}}{\lambda_{e}} \times \frac{A r^{40 *}}{K^{40}}+1\right]:$

$K^{40} / K=1.22 \times 10^{-4} \mathrm{~g} . / \mathrm{g}$.

Note: $A r^{40 *}$ refers to radiogenic $A r^{40}$.

$M . Y$. refers to millions of vears. 


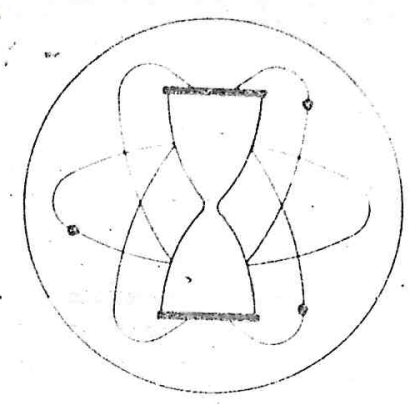

\section{KRUEGER ENTERPRISES, INC. Geochron Laboratories Division}

24 BLACKSTONE STREET O CAMBRIDGE, MA. $02139 \cdot(617) \cdot 876.3691$

POTASSIUM-ARGON AGE DETERMMNATION

REPORT OF ANALYTICAL WORK

Our Sample No. R-2170

Date Received: 29 November 1971

Vour Feference: ICF-?

Date Reported: 4 January 1972

Submitiod by: Drane Ho Reno

Amoco Production Co.

Security Life Bldg.

Denver, Colorado 80202

Sample Description \& Locality:

Sheared basalt (?)

Northern Alaska

Material Analyzed: Whole rock, crushed to $-40 /+100$ mesh.
$\operatorname{Ar}^{40 * / K^{40}}=.01729$
$\mathrm{AGE}=275 \pm 11$
M.Y.

Argon Analyses:

$\begin{array}{ccc}\operatorname{Ar}^{40 *}, \text { ppm. } & \operatorname{Ar}^{40 *} / \operatorname{rotal}^{40}{ }^{40} & \text { Ave. Ar }{ }^{40 *}, \text { ppm. } \\ .02756 & .827 & .02709 \\ .02662 & .679 & \end{array}$

Potassium Analyses:

$\% K$

1.278

1.290

Ave. $\%$ K

1.284

$K^{40}, p p m$

1.566

\section{Constants Used:}

$\lambda_{\beta}=4.72 \times 10^{-10} /$ year

$\lambda_{\mathrm{e}}=0.585 \times 10^{-10} /$ year

$A G E=\frac{1}{\lambda_{e}+\lambda_{\beta}} \ln \left[\frac{\lambda_{\beta}+\lambda_{e}}{\lambda_{e}} \times \frac{A r^{40 *}}{K^{40}}+1\right]$

$K^{40} / K=1.22 \times 10^{-4} \mathrm{~g} / \mathrm{g}$.

Note: $\mathrm{Ar}^{40} *$ refers to radiogenic $\mathrm{Ar}^{40}$.

$M . Y$. refers to millions of years. 


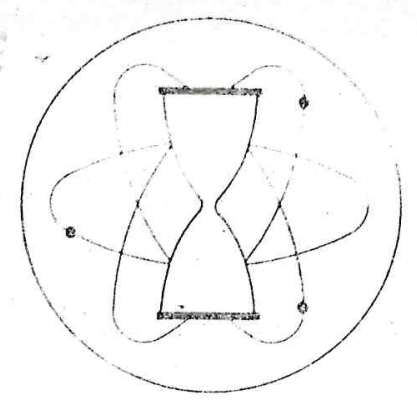

\section{KRUEGER ENTERPRISES, INC. GEOCHRON LABORATORIES DIVISION}

24 BLACKSTONE STREET O CAMBRIDGE, MA. 02139. (617).876.3691

Our Sampla No. R-2169

Date Received: 29 Novernber 1971

Your Reference: LCF- 19

Date Reported: 4 January 1972

Submitted by: Duare II. Rexo

Amoco Production Co.

Security Iife Bldg。

Derver, Colorado 80202

Sample Description \& Locality:

Basalt

Worthern Alaska

Material Analyzed: Whoje rock, crushed to $-40 /+100$ mesh.

Ar $40 * / K^{20}=: 001153$

$A G E=$

$19.6 \pm 1.0$

M.Y.

Argon Analyses:

Ar $40 \%$, ppm.

.002059

.001934
$\operatorname{Ar}^{40 * / T o t a l ~ A r}{ }^{40}$

.188

.168
Ave. Ar $40 *$, ppm.

.001997

Potassium Analyses:

$\% k$

1.428

1.411
Ave. \%k

1.419
$K^{40}, \mathrm{ppm}$

1.731

\section{Constants Used:}

$\lambda_{\beta}=4.72 \times 10^{-10} /$ year

$\lambda_{e}=0.585 \times 10^{-10} /$ year

$A G E=\frac{1}{\lambda_{e}+\lambda_{\hat{\beta}}} \ln \left[\frac{\lambda_{\beta}+\lambda_{e}}{\lambda_{e}} \times \frac{A r^{40 *}}{K^{40}}+1\right]$

$K^{40} / K=1.22 \times 10^{-4} \mathrm{~g} . / \mathrm{g}$.

Note: $\mathrm{Ar}^{40 *}$ refers to radiogenic $\mathrm{Ar}^{40}$.

M.V. refers to millions of years. 